\title{
Impaired prosodic discrimination in patients with schizophrenia
} Derek Tracy*

\author{
Address: The Institute of Psychiatry, London, UK \\ * Corresponding author
}

\author{
from International Society on Brain and Behaviour: 2nd International Congress on Brain and Behaviour \\ Thessaloniki, Greece. 17-20 November 2005 \\ Published: 28 February 2006 \\ Annals of General Psychiatry 2006, 5(Suppl I):S209 doi:I0.II86/I744-859X-5-SI-S209
}

\section{Background}

Auditory verbal hallucinations (AVH) and persecutory delusions are cardinal features of schizophrenia, though their pathophysiology is uncleaar. A contempory model proposes that AVH occur because self-generated inner speech is misperceived as externally generated, and delusions may develop secondarily [1]. Recent neuroimaging studies suggest that both speech generation and perception areas are activated during AVH [2]. Prosodic aspects of language have been demonstrated to be abnormal in patients with schizophrenia [3], but such work focused upon the affective aspects of prosody, with little investigation of the more elementary processing of speech perception such as pitch and temporal pattern. Prosodic information may be necessary for accurate verbal selfmonitoring, and its disturbance may contribute to the aetiology of psychotic symptoms. This study examined prosodic discrimination in patients with schizophrenia compared to normal controls.

\section{Materials and methods}

Fifteen subjects with schizophrenia and fifteen volunteers without any history of mental illness underwent a music and prosody discrimination task of lexically matched sentence pairs and their musical, non-verbal, analogues. Variations occurred in three attributes in these pairs; terminal-pitch pattern, internal pitch pattern and timing shift. Subjects abilities to distinguish differences in these categories were measured.

\section{Results}

Patients showed a significant reduction in the accuracy of the internal pitch discrimination compared with controls in both sentence and musical pairs. There were no differences in detecting terminal pitch changes or timing shift in either sentences or musical pairs.

\section{Discussion}

These findings suggest that patients with schizophrenia have a deficit in discriminating fundamental acoustic features underlying the internal pattern of prosodic and music perception. Such internal pitch changes place greater efforts on working memory than terminal pitch changes, suggesting a potential role for working memory; however, there were not significant deficits in timing shift, which indicates that memory may be contibutory, but not a fundamental cause of the deficit. Correlation analysis showed that the disturbance of internal pitch discrimination of the verbal prosodic sentences was associated with the presence of positive sypmtoms of schizophrenia, suggesting that a specific deficit in prosodic comprehension may contribute to symptom aetiology.

\section{References}

I. Frith $C D$, Done DJ: Towards a neuropsychology of schizophrenia. British Journal of Psychiatry 1988, 153:437-443.

2. Murphy D, Cutting J: Prosodic comprehension and expression in schizophrenia. Journal of Neurology, Neurosurgery and Psychiatry 1990, 53:727-730.

3. Shergill SS, Brammer MJ, Williams SC, Murray RM, McGuire PK: Mapping auditory hallucinations in schizophrenia using functional magnetic resonance imaging. Archives of General Psychiatry 2000, 57:1033-1038.

4. Buchanan TW, Lutz K, Mirazazade S, Specht K, Shah NJ, Zilles K, jancke $L$ : Recognition of emotional prosody and verbal components of spoken language: an fMRI study. Cognitive Brain Research 2000, 9:227-238. 